\title{
Médiation Culturelle en Traduction : l'importance du rôle du traducteur
}

\author{
Ana Maria Simão Saldanha \\ Prof. Adjunta Convidada (Instituto Politécnico De Macau) \\ anasaldanha2@gmail.com
}

RÉSUMÉ: Par le présent article, nous prétendons réfléchir sur l'acte de traduction, entendu comme un processus de transfert culturel réalisé grâce à la médiation d'un traducteur. Suivant les propositions d'Umberto Eco et de Susan Bassnett, nous nous pencherons sur l'acte de traduction qui s'établi entre deux codes linguistiques différents (traduction interlinguistique), lequel exige une compréhension approfondie de deux cultures et pourtant des connaissances et des compétences qui vont au-delà des connaissances et des compétences linguistiques.

Mots-clés: médiation culturelle; traduction; compétences en traduction; traductologie.

\section{Cultural Mediation in Translation: the role of the translator}

ABSTRACT: In this article, we aim to reflect on the act of translation, understood as a process of cultural transfer carried out thanks to the mediation of a translator. Following the proposals of Umberto Eco and Susan Bassnett, we will focus on the act of translation which is established between two different linguistic codes (interlinguistic translation), which requires a deep understanding of two cultures and yet knowledge and skills that go beyond language knowledge and skills.

Keywords: cultural mediation; translation; translation skills; traductology. 


\section{Introduction}

Nous nous proposons de réfléchir sur l'acte de traduction et la médiation culturelle inhérente à cet acte. Partant de la division classique que Roman Jakobson (1963 [1959]) a proposée pour les différents processus de transition linguistique (intralinguistique, interlinguistique et intersémiotique), et en poursuivant avec les travaux développées par Paulo Rónai (1976), Umberto Eco (2007), Octavio Paz (2009), Susan Bassnet (2003), entre autres, nous considèrerons la traduction comme un processus de transition qui s'opère soit entre différents codes linguistiques et culturels, soit au sein d'un même code linguistique et culturel. En raison des limites qu'un article scientifique naturellement nous impose, nous concentrerons le présent travail sur le processus de traduction interlinguistique et, surtout, sur le rôle du traducteur en tant que médiateur entre deux cultures ${ }^{1}$.

Nous poursuivrons une approche interdisciplinaire dont le point de départ remonte à 1923, lorsque Walter Benjamin proposa une pratique interdisciplinaire pour l'étude et la pratique de la traduction, en utilisant des contributions des disciplines les plus variées. Considérant que la traduction constitue un passage entre deux cultures, nous poursuivrons notre réflexion sur la médiation culturelle et la traduction en nous appuyant sur les approches théoriques qui estiment que le traducteur ne doit pas avoir en considération que des règles strictement linguistiques, mais aussi des éléments culturels permettant de comprendre le texte source.

Dans ce sens, le processus de traduction interlinguistique n'est pas seulement un acte de transfert entre deux langues différentes, car il assume également un rôle important dans l'intermédiation entre différents textes et différentes cultures. Dans ce processus, le traducteur apparaît comme un médiateur, dont les options de traduction résultent d'une capacité de négociation constante de la solution qui lui semble la plus appropriée. Comme le prévenait Eco (2007), une traduction implique que la complicité et l'engagement soient des éléments cruciaux pour la qualité du produit final.

Dans un premier temps, nous nous occuperons de comprendre l'acte de traduire, nous accompagnerons après les différentes approches théoriques, pour finalement comprendre l'acte de traduction comme une médiation culturelle.

\section{Qu'est-ce que la traduction?}

Étymologiquement, traduire remonte au verbe latin traducere, c'est-à-dire, conduire au-delà ou transfert/transférer. Aujourd'hui, transférer est l'équivalent

\footnotetext{
${ }^{1}$ Malgré cela, nous n'oublierons pas le constant dialogue et la constante proximité entre l'acte de traduction intralinguistique (interprétation des signes verbaux, à travers d'autres signes de la même langue) et l'acte de traduction entre deux langues différentes, tous les deux impliquant, d'ailleurs, la compréhension de trois facteurs distincts: le texte source, le traducteur et le texte cible.
} 
de transposer, de révéler, d'expliquer, de manifester, de représenter ou de symboliser. Ainsi, nous pouvons regarder la traduction comme une opération de transfert ou de transposition d'une langue à une autre. De manière générale, traduire est donc toute opération de transfert, soit entre différents systèmes sémiotiques, soit au sein d'un même système sémiotique.

L'acte de traduction dans une même langue - opération linguistique connu comme paraphrase - est appelé par Roman Jakobson (1963 [1959]) de traduction intralinguistique. Octavio Paz (2009), de son côté, la caractérise de la façon suivante :

Apprendre à parler, c'est apprendre à traduire : quand un enfant demande à sa mère le sens de tel ou tel mot, ce qu'il demande vraiment c'est que le mot inconnu soit traduit dans sa langue. La traduction au sein d'une langue n'est pas, en ce sens, essentiellement différente de la traduction entre deux langues, et l'histoire de tous les peuples répète l'expérience de l'enfance (p. 9)².

En faisant la division classique des différents types de traduction (intralinguistique, interlinguistique et intersémiotique), Roman Jakobson (1963 [1959]) explique que la traduction intralinguistique résulte de l'interprétation des signes verbaux grâce à d'autres signes de la même langue. Or, c'est cette traduction opérée à l'intérieur du même code linguistique qu'Octavio Paz (2009) compare à l'expérience enfantine d'apprentissage de la parole. Les deux auteurs semblent ainsi rapprocher la traduction intralinguistique de la traduction interlinguistique.

Selon cette approche, il n'y aurait pas d'activité linguistique sans traduction. Ainsi, l'apprentissage d'une langue impliquerait nécessairement la concrétisation d'un acte de traduction.

Susan Bassnett (2003) nous rappelle qu'autrefois la traduction était considérée comme une activité marginale. La théoricienne de la traduction rappelle encore que ce n'est qu'au $X X^{e}$ siècle que la traduction a été considérée comme un acte fondamental d'échange entre les êtres humains. Paulo Rónai (1976), de son côté, a contesté les définitions qui jusqu'alors avaient été attribués au mot traduction:

En définissant traduction, les dictionnaires (...) se limitent à dire que «traduire, c'est passer à une autre langue ». Cependant, la comparaison la plus évidente est fournie par l'étymologie : en latin, tranducere c'est prendre quelqu'un par la main vers l'autre côté, vers un autre endroit. Le sujet de ce verbe est le traducteur, l'objet direct, l'auteur de l'original à qui le traducteur introduit dans un

\footnotetext{
${ }^{2}$ Notre Traduction.
} 
nouvel environnement [...] Mais l'image peut être aussi comprise $\mathrm{d}^{\prime}$ une autre manière : le traducteur prend la main du lecteur pour l'emmener vers un support linguistique autre que le sien (p. 3-4) ${ }^{3}$.

Paulo Rónai (1976) définit comme traduction naturalisatrice la traduction qui « conduit une œuvre étrangère dans un autre environnement linguistique, en l'adaptant autant que possible aux coutumes du nouvel environnement, en supprimant ses caractéristiques exotiques et en nous faisant oublier qu'elle reflète une réalité éloignée et essentiellement différente (p. 2-3) ${ }^{4}$. Au contraire, l'auteur définit comme traduction identificatrice celle qui « conduit le lecteur dans le pays de l'œuvre qu'il lit et qui conserve soigneusement ce qui est étrange, authentique, accentuant à chaque instant son origine étrangère » (p. 3-4 $)^{5}$. Lawrence Venuti (1995), de son côté, parle de domestication et d'étrangéisation, attirant l'attention sur le fait que l'idéologie et le discours dominant déterminent généralement les stratégies de traduction et la suppression du traducteur. Venuti parle alors de l'invisibilité du rôle du traducteur.

Dans son essai Les Tours de Babel, Jacques Derrida (1985) évoque le texte biblique et soutient que Dieu a séparé les hommes pour créer la traduction. Derrida (1985) rappelle que selon la confusion babélique décrite par la Bible (Genèse 11), la terre entière avait une seule langue et utilisait les mêmes mots. Cependant, un jour, quelques hommes partant pour l'est ont trouvé dans le pays de Senar une plaine où ils se sont installés. Ici, ils ont décidé de construire une ville et une tour dont le sommet devrait atteindre le ciel. Suite à cet évènement, le Seigneur a voulu freiner l'ambition des hommes, attribuant à chaque peuple une langue différente et la construction de la ville a cessé :

L'architecture renverse l'image négative de la tour de Babel en s'inspirant de ce mythe pour nourrir des utopies architecturales et urbaines. En effet, elle symbolise la ville cosmopolite composée de gens qui ne se comprennent pas mais qui se complètent, le but étant de socialiser et d'humaniser la ville. Les métropoles concentrent alors les pouvoirs politiques, économiques, mais aussi les populations nouvelles et les influences culturelles de tous horizons. $C^{\prime}$ est dans ce cadre que des transformations urbaines majeures et de nouveaux projets urbains utopiques fleurissent. La punition évoquée dans la genèse a été largement dépassée par les hommes qui ne cessent de vouloir se rassembler et créer des mixités sociales (DERRIDA, 1985, p. 211).

\footnotetext{
${ }^{3}$ Notre Traduction.

${ }^{4}$ Notre Traduction.

${ }^{5}$ Notre Traduction.
} 
George Steiner (1998) considère que le fait que des milliers de langues différentes et mutuellement incompréhensibles soient parlées sur toute la planète est une expression claire de l'énigme profonde de l'individualité humaine, étant la preuve biogénétique et biosociale qu'il n'y a pas deux êtres humains entièrement identiques. Étant donné que les êtres humains parlent différentes langues, Steiner signale que la traduction est un besoin humain présent dans différentes situations, pouvant varier selon le sexe, la classe sociale et le groupe d'âge.

\section{Les différentes approches de la traduction}

Tout au long de l'Histoire de l'Humanité, divers auteurs ont réfléchi sur l'acte de traduire. En Occident, ce serait lors de la Renaissance - notamment avec le besoin d'imposer la parole des religions universelles - que la traduction revêtira une importance particulière.

Etienne Dolet $(1830 \text { [1544] })^{6}$ était un humaniste français que considérait que la traduction devrait s'appuyer sur cinq principes: le traducteur doit connaître parfaitement le sens et le sujet à traduire; le traducteur doit connaître parfaitement la langue de l'auteur qu'il traduit; le traducteur doit maîtriser la langue vers laquelle il a l'intention de traduire; le traducteur ne doit pas traduire mot à mot; le traducteur doit utiliser des mots couramment utilisés; le traducteur doit observer l'harmonie du discours. Déjà au XVIe siècle, Etienne Dolet signalait donc l'importance de comprendre le texte source ainsi que le fait que le traducteur est plus qu'un linguiste compétent et que la traduction impliquait une approximation au texte source. Pour cela il a été d'ailleurs condamné par l'Inquisition. En effet, "L'acte d'accusation tient sa preuve d'une "mauvaise" traduction d'un dialogue erronément attribué à Platon, l'Axiochus (...)». L'erreur de Dolet se situerait dans trois mots en trop (« rien du tout ») dans un argument visant à montrer que la mort n' est rien (« après la mort, tu ne seras plus rien du tout ») et qui serait la preuve que Dolet ne croyait pas - ou ferait croire que Platon ne croyait pas - en l'immortalité de l'âme après la mort » (LEMIEUX, 2016).

John Dryden (2017 [1680]), dans la préface aux Épitres d'Ovide, signale l'existence de trois types de traduction : la métaphrase, c'est-à-dire « la traduction d'un auteur mot à mot, et vers par vers, d'une langue vers l'autre », la paraphrase, c'est-à-dire la traduction du sens, "traduction avec latitude, où le traducteur garde les yeux sur son auteur, de manière à ne jamais le perdre de vue », et l'imitation où le traducteur «se donne la liberté, non seulement de s'éloigner des mots et du sens, mais encore de les délaisser tous deux quand il en voit l'occasion, et de tirer seulement de l'original quelque inspiration générale

\footnotetext{
${ }^{6}$ Etienne Dolet : 1509 (n) -1546 (m.).
} 
pour composer sur le thème les variations qu'il lui plaira ». Alexander Fraser Tytler (1904 [1791]), de son côté, dans Les principes de la traduction, défend qu'un «traducteur poétique a le devoir de ne jamais laisser tomber son original ${ }^{7}$ ( $\mathrm{p}$. 45). Il énumère alors trois principes de la traduction : la traduction doit faire une transcription complète de l'idée de l'œuvre originale, du style et de la manière d'écrire de l'auteur et doit conserver le caractère naturel de l'original. Friedrich Schleiermacher, dans son œuvre Sur les différentes méthodes de traduction (2001 [1813]) soutien que la traduction apparaît comme une entreprise un peu folle. C'est pourquoi, dit Schleiermacher, « désespérant d'atteindre ce but, ou, si l'on veut, avant même d'être parvenu à le penser clairement, on a inventé, non par véritable sens de l'art de la langue, mais par nécessité spirituelle et par habileté intellectuelle, deux autres manières de connaître les œuvres des langues étrangères, qui tantôt se débarrassent violemment de ces difficultés, tantôt les contournent, mais en abandonnant complètement l'idée de la traduction ici proposée; ce sont la paraphrase et l'imitation » (p. 31). Il propose alors deux stratégies de traduction : déplacer l'auteur vers le lecteur ou déplacer le lecteur vers l'auteur.

Dans le sens de comprendre l'évolution des théories de la traduction, George Steiner (1998) va proposer différentes étapes chronologiques. Ainsi, la première étape débute avec les propositions de Cicero et s'étend jusqu'en 1804. Cette première étape de l'Histoire de la traduction abrite donc les théories proposées par Saint Jérôme, Leonardo Bruni, Montaigne, Dryden, entre autres. Il s'ensuit une étape lors de laquelle le problème de la traduction est envisagé d'un point de vue plus philosophique. Les auteurs s'appuient alors sur des propositions théoriques et sur des investigations herméneutiques (Tytler, Schlegel, Schleiermacher et Humboldt). Nous arrivons finalement aux courant modernes qui, toujours selon Steiner (1998), ne débutent que dans les années 1940.

De signaler cependant l'œuvre majeur de Walter Benjamin, La tâche du traducteur, publiée en 1923, quand effectivement les études herméneutiques sur la traduction et l'interprétation gagnent une nouvelle vie. La théorie et la pratique de la traduction deviennent alors un exercice interdisciplinaire où différentes disciplines interagissent (psychologie, anthropologie, sociologie, ethnographie, littérature comparée, sociolinguistique). Sur la traduction, défend alors l'auteur :

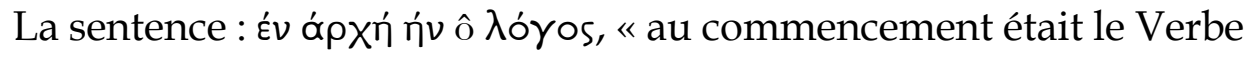
», vaut aussi pour la traduction. Elle a le droit ou même le devoir, en face du sens, de laisser aller sa langue, afin de ne pas faire sonner son propre mode d'intention comme restitution de l'intention de

\footnotetext{
${ }^{7}$ Notre Traduction.
} 
celui-ci, mais comme harmonique, comme complément à la langue où celle-ci se communique. C'est pourquoi, surtout à l'époque où elle paraît, le plus grand éloge qu'on puisse faire à une traduction n'est pas qu'on la lise comme un original de sa langue. Cette fidélité dont la littéralité est caution signifie bien plutôt qu'elle laisse exprimer à l'œuvre sa grande nostalgie d'un complément de sa langue. La vraie traduction est transparente, elle ne cache pas l'original, n'offusque pas sa lumière, mais c'est la pure langue, comme renforcée par son propre médium, qu'elle fait tomber d'autant plus pleinement sur l'original (p. 156).

Nous pouvons donc conclure que, grosso modo, les théories de la traduction ont proposés trois grandes stratégies distinctes: la traduction littérale, la traduction intermédiaire (l'énoncé traduit cherche à être fidèle et, malgré cela, autonome) et la traduction comme interprétation.

Tout au long du XXe siècle, de nombreuses tendances se sont alors développées, notamment depuis que la Traduction a eu une reconnaissance académique. C'est ainsi que se sont développés les théories sur l'équivalence des langues, les théories fonctionnalistes (notamment grâce à Christiane Nord), les approches discursives, les études descriptives et normatives, la théorie des polysystèmes (d'Itamar Even-Zohar), les études de corpus, les études culturelles et les approches interdisciplinaires.

L'un des plus grands clivages était la controverse entre la traduction libre et la traduction littérale. À ce propos, Paulo Rónai (1976) affirme que, généralement, 1 'on considère « que la traduction fidèle est la traduction littérale, et donc, toute traduction qui n'est pas littérale est libre » (p. 20) ${ }^{8}$. Par contre, signale le même auteur, l'existence de la traduction littérale ne pourrait être possible que s'il existait des langues suffisamment similaires pour permettre au traducteur de se limiter à une simple transposition de mots ou d'expressions d'une langue à une autre. Cependant,

il n'y a pas de langues comme ça, pas même parmi les langues apparentées. Les innombrables différences structurelles entre la langue d'origine et la traduction obligent le traducteur à choisir entre deux ou plusieurs solutions à la fois et, dans son choix, il est constamment inspiré par l'esprit de la langue vers laquelle il traduit (RÓNAI, 1976, p. 21) ${ }^{9}$.

\footnotetext{
${ }^{8}$ Notre Traduction.

${ }^{9}$ Notre Traduction.
} 
Sur la question de la fidélité, Umberto Eco (2007) signale qu'il n'est pas possible d'être complètement fidèle, puisque lors d'une traduction on ne dit jamais exactement la même chose, mais presque la même chose :

La soi-disante « fidélité » des traductions n'est pas un critère qui conduit à la seule traduction acceptable [...]. Au contraire, la fidélité est la tendance à croire que la traduction est toujours possible si le texte source a été interprété avec une complicité passionnée, c'est aussi l'engagement d'identifier ce qui, pour nous, constitue le sens profond d'un texte ainsi que la capacité de négocier à chaque instant la solution qui nous semble la plus juste (p. 426) ${ }^{10}$.

Si la question de la fidélité en traduction a fait l'objet d'une profonde réflexion de la part de divers auteurs au fil du temps (comme Cicéron, Horace, Schleiermacher, Dryden, Dolet, Venuti ou Steiner), ce n'est cependant que tardivement que les réflexions et les théories sur la fidélité en traduction se sont éloignées de la traduction mot à mot. Ainsi, comme le signale Munday (2001), il a fallu atteindre la fin du XVIIe siècle pour que la fidélité s'identifie généralement au sens plutôt qu'aux paroles de l'auteur.

Le concept de fidélité en traduction a donc changé avec le temps et aujourd'hui nous pouvons dire que le traducteur a le devoir de rester pas seulement fidèle, mais aussi innovateur. Si, comme l'a démontré Steiner (1998), le traducteur doit rester fidèle à son texte source et la fidélité doit rester un critère essentiel pour tout traducteur, cela ne veut pas dire que le traducteur doit faire une traduction littérale ou mot à mot, puisque sa capacité de négociation et de création doit aussi rester un vecteur d'importance lors de son travail.

\section{Le transfert entre deux cultures distinctes}

La traduction est donc un terme à des multiples facettes et plusieurs sont les auteurs qui essaient de lui attribuer une conceptualisation précise. Jeremy Munday (2001) signale que cette diversité de points de vue et de conceptualisations est aussi présente lors de l'examen des aspects généraux du domaine de la traduction - le produit (le texte traduit) et le processus (l'acte de traduire et la traduction elle-même).

Susan Bassnett (2003) considère que la traduction n'est pas seulement le transfert de textes d'une langue à une autre, mais un processus de négociation qui s'opère entre des textes et des cultures et dans lequel les transactions négociées par un traducteur ont lieu. C'est aussi dans ce sens qui va la réflexion d'Umberto Eco (2007) :

\footnotetext{
${ }^{10}$ Notre Traduction.
} 
une traduction n'est pas seulement un passage entre deux langues, mais entre deux cultures ou deux encyclopédies. Un traducteur doit non seulement tenir compte des règles strictement linguistiques, mais aussi des éléments culturels, au sens large du terme (p. 190 $)^{11}$.

Or, la traduction interlinguistique comprend trois éléments fondamentaux : le texte source, le traducteur et le texte d'arrivée. Le traducteur effectue alors une opération dans laquelle il agit en tant que lecteur et interprète, puisque c' est grâce à un processus de lecture et d'interprétation d'un texte source que le traducteur produit un texte d'arrivée dans un certain code culturel et linguistique. Selon Jakobson (1963 [1959]) :

au niveau de la traduction interlinguistique, il n'y a généralement pas d'équivalence complète entre les unités de code, tandis que les messages peuvent servir d'interprétation adéquate des unités de code ou des messages étrangers [...]. Plus souvent, cependant, lors de la traduction d'une langue vers une autre, les messages de l'une des langues sont remplacés, non par des unités de code distinctes, mais par des messages entiers dans une autre langue. Une telle traduction est une forme de parole indirecte : le traducteur recode et transmet un message reçu d'une source. Ainsi, la traduction implique deux messages équivalents dans deux codes différents ( $p$. $65)^{12}$.

Le traducteur interlinguistique a donc toujours contribué à l'urgence, à l'enrichissement et à la promotion des langues et des littératures nationales. Il a donc toujours contribué à l'éveil d'une conscience collective des groupes ethniques et linguistiques, promouvant et préservant un certain patrimoine culturel. C'est pourquoi Paulo Rónai (1976) signale qu'une connaissance approximative de la langue de l'auteur dont l'œuvre est en train d'être traduite n'est pas suffisante. En effet, le traducteur doit connaître intimement la langue $\mathrm{d}^{\prime}$ origine afin de capturer, en plus du contenu strictement logique, le ton exact, les effets indirects ainsi que les intentions cachées de l'auteur. La fidélité, comme nous l'avons signalé précédemment, n'est donc pas obtenue par une traduction littérale, mais plutôt par un remplacement continu. En ce sens, l'art du traducteur est précisément de savoir quand est-ce qu'il doit chercher des équivalences. Vu qu'il n'y a pas d'équivalences absolues, un mot, une phrase ou une phrase de l'original peuvent être souvent transporté(e)s/traduit(e)s de différentes manières, raison pour laquelle il est difficile de dire avec fermeté quelle est la meilleure traduction.

\footnotetext{
${ }^{11}$ Notre Traduction.

12 Notre Traduction.
} 
Le traducteur est donc un médiateur. C'est pourquoi Roman Jakobson (1963 [1959]) le considère comme un (re)codificateur ou transmetteur d'un message: bien que différents codes soient utilisés, la traduction implique nécessairement des messages équivalents. D'après Paulo Rónai (1976), l'acte de transposer d'une place à une autre, proposé par le verbe latin traducere, peut aujourd'hui être comparé au produit final d'un processus de traduction, puisque le traducteur permet de conduire le lecteur dans un environnement linguistique et culturel qui lui est étranger.

\section{La médiation culturelle : rôle du traducteur}

Depuis l'œuvre de Georges Steiner, After Babel: Aspects of Language and Translation, la traduction est un acte de communication. Malgré cela, les approches théoriques sur l'acte de traduction ne sont pas toutes d'accord par rapport à la pertinence des différences culturelles sous-jacentes à cet acte.

L'un des domaines de controverse concerne la définition de la culture ellemême. En effet, il existe une division historique entre ceux qui perçoivent la langue et la culture comme deux entités distinctes et ceux qui considèrent la langue comme faisant partie d'une culture. Dans le premier cas, la traduction est vue comme une activité linguistique d'encodage et de décodage universaliste «transferring meaning from the SL to the TL, using what Reddy (1973/1993) called the 'conduit metaphor of language transference'. Here, culture and any cultural differences can be carried by the language without significant loss » (KATAN, 2009, p. 75), tandis que d'autres théoriciens, comme Nida (2002), estiment que " the context actually provides more distinction of meaning than the term being analyzed » (apud KATAN, p. 29). Christiane Nord (1997), de son côté, considère que la caractérisation de culture peut se comprendre en suivant la définition de l'ethnologue américain Goodenough (1964 apud Nord, 1997):

As I see it, a society's culture consists of whatever it is one has to know or believe in order to operate in a manner acceptable to its members, and do so in any role that they accept for any one of themselves. Culture, being what people have to learn as distinct from their biological heritage, must consist of the end product of learning: knowledge, in a most general, if relative, sense of the term. By this definition, we should note that culture is not a material phenomenon; it does not consist of things, people, behaviour, or emotions. It is rather an organisation of these things. It is the forms of things that people have in mind, their models for perceiving, relating, and otherwise interpreting them (p. 100).

Chaque aspect de la culture est ainsi lié dans un système plus élargi qui permet de former un contexte unificateur d'une culture donnée. Christian Nord 
(1997) soutient alors que la traduction est un acte de comparaison entre cultures et que les éléments linguistiques du texte cible doivent être sélectionnés de façon à faire correspondre l'effet du texte cible à ses fonctions. Le traducteur doit alors décider s'il souhaite imiter le style du texte source et, dans ce cas, il doit déterminer quel serait le moyen le plus approprié pour atteindre la fonction du texte.

Vermeer (1998), de son côté, se réfère à la traduction comme « a skoposorientated cultural process » (p. 56), tandis que Venuti (2000) remarque que la traduction est « a linguistic "zone of contact" between the foreign and translating cultures » (p. 477) et rajoute que les traductions, en particulier celles de textes étrangers ayant fait l'objet d'une diffusion massive, favorisent la création des communautés de lecteurs qui, dans des circonstances ordinaires, seraient séparées de la culture/lecture d'un texte source par des différences culturelles et des divisions sociales.

Toury (1995), de son côté, soutient que les traductions sont en fait initiées par la culture cible qui est en manque de quelque chose qui n'existe que dans la culture du texte source. Ainsi, afin de fournir à la culture cible ce qui lui manque, le traducteur doit être un expert biculturel et employer les stratégies de traduction nécessaires pour que le transfert culturel puisse s'opérer.

Nous pouvons alors conclure que le texte cible est un instrument de communication qui doit fonctionner de façon optimale dans la culture cible. Le traducteur assume alors le rôle de médiateur, devant être un expert en communication interculturelle, travailler selon une stratégie de traduction planifiée et structurée et être capable d'embrasser toutes les différences culturelles impliquées dans la construction d'un document dans une autre culture.

\section{Conclusion}

Tout au long du XXe siècle, la traduction a eu diverses approches théoriques et conceptuelles, soit par rapport aux produits de la traduction, soit par rapport aux processus de traduction.

Suivant une approche interdisciplinaire (dont le point de départ remonte à 1923, lorsque Walter Benjamin propose une démarche interdisciplinaire pour l'étude et la pratique de la traduction) et dans la suite des travaux développés par des approches théoriques récentes, nous considérons qu'une traduction constitue un passage entre deux cultures. C'est d'ailleurs pourquoi le traducteur doit non seulement avoir en considération des règles strictement linguistiques, mais aussi des éléments culturels, lesquels sont prépondérants à la compréhension du texte source.

Le processus de traduction interlinguistique n'est donc pas seulement un acte de transfert entre deux langues différentes, mais aussi un processus 
d'intermédiation entre des textes et des cultures où le traducteur apparaît comme un médiateur; la traduction n'est donc pas une simple transposition de mots ou d'expressions entre deux langues.

Ayant plusieurs options de traduction à sa disposition, le choix du traducteur est réalisé selon la subjectivité, la connaissance de l'œuvre, de l'auteur traduit, de l'environnement socioculturel de l'auteur (au sens large) et des éléments socio-historiques. Comme le déclare Eco (2007), le choix du traducteur est donc le résultat de compétences de négociation constantes qui dépendent des compétences et des connaissances socio-culturelles de la solution qui lui semble la plus appropriée: la complicité et l'engagement sont donc des éléments cruciaux pour la qualité du produit final et, par conséquent, pour la compétence traductrice.

\section{BIBLIOGRAPHIE}

BASSNETT, Susan. Estudos da tradução. Lisboa : Fundação Calouste Gulbenkian, 2003. Traduit par Vivina de Campos Figueiredo.

BENJAMIN, Walter. La tâche du traducteur, Poésie, nº 55, pp. 150-158, 1991 [1923]. Traduit par Martine Broda.

DERRIDA, Jacques. Des tours de Babel. In : GRAHAM, Joseph (ed.). Difference in translation. Éditions Cornell University Press, pp. 209-248, 1985.

DOLET, Étienne [Estienne]. Le Second Enfer. Paris : Techener, 1830 [Lyon, 1544]. En ligne : <http:/ / dolet.editionsartulis.fr/> (consulté le 12 janvier 2020).

DRYDEN, John. Préface aux Épitres d'Ovide. Montréal : Université de Montréal, 2017 [1680].. Traduction et édition: Marie-Alice Belle. En ligne : <https:/ / ttt.hypotheses.org/159> (consulté le 14/02/2020).

ECO, Umberto. Quase a mesma coisa. Experiências de tradução. Rio de Janeiro/São Paulo : Record, 2007. Traduit par Eliana Aguiar.

JAKOBSON, Roman. Aspectos linguísticos da tradução. In: Linguística e Comunicação. São Paulo, Editora Cultrix, pp. 63-82, 1963 [1959].

KATAN, David. Translation as intercultural communication. In Jeremy Munday. The Routledge companion to translation studies. London/New York, Routledge, pp. 74-92, 2009. 
LEMIEUX, René. Thanatographie d'Étienne Dolet : spéculer sur la liberté en traduction, la Modernité et la crainte de mourir, Cygne noir, $n^{\circ} 4$, 2016. En ligne : $\quad$ <http://revuecygnenoir.org/numero/article/dolet-liberte-traduction> (consulté le 23/01/2020).

MUNDAY, Jeremy. Introducing Translation Studies. London/New York : Routledge, 2001.

NIDA, Eugene Albert. Contexts in Translating. Amsterdam and Philadelphia: John Benjamins, 2002.

NORD, Christian. Translating as a purposeful activity: functionalist approaches explained. Manchester: St Jerome, 1997.

PAZ, Octavio . Tradução, literatura e literalidade. Belo Horizonte: FALE/UFMG, 2009. Traduit par Doralice Alves de Queiroz.

RÓNAI, Paulo. A tradução vivida. Rio de Janeiro: EDUCOM, 1976.

SCHLEIERMACHER, Friedrich. Sobre os diferentes métodos de tradução . In: Clássicos da teoria da tradução. Florianópolis, UFSC, pp. 26-87, 2001 [1813]. Traduit par Margarete von Mühlen Poll.

STEINER, George. After Babel: Aspects of Language and Translation. Oxford: OUP, 1998.

TOURY, Georges. Descriptive translation studies and beyond. Amsterdam: Benjamins, 1995.

TYTLER, Alexander Fraser. Les principes de la traduction. Londres: J.M. Dent \& Sons Ltd. / New York: E. P. Dutton \& Co. Inc., 1904 [1791]. En ligne : $<$ https:// bibdig.biblioteca.unesp.br/bitstream/handle/10/6622/essay-on-theprinciples-of-translation.pdf?sequence $=3 \&$ \&isAllowed $=y>\quad$ (consulté le 14/02/2020).

VENUTI, Lawrence. The translator's invisibility: a history of translation. London: Routledge, 1995. 
VENUTI, Lawrence. Translation, community, utopia. In: Venuti Lawrence (ed). The translation studies reader. London, Routledge, pp. 468-488, 2000.

VERMEER, Hans Josef. Starting to unask what translatology is about, Target, $\mathrm{n}^{\circ}$ 10(1), pp. 41-68, 1998.

Data de envio: 23/05/2020

Data de aprovação: 21/09/2020

Data de publicação: 21/12/2020 